\title{
Immunoglobulins in paired specimens of vitreous and subretinal fluids from patients with rhegmatogenous retinal detachment
}

\author{
Geoffrey E Rose, Brenda M Billington, A H Chignell
}

\begin{abstract}
Evidence suggests that there is a net movement of fluid through the retinal break in eyes with rhegmatogenous retinal detachment, this net movement being directed from the vitreous humour into the subretinal space. However, it remains uncertain how much fluid exchange occurs in both directions across such breaks. The concentration ratios of $\mathbf{I g G / I g M}$ or $\operatorname{IgA} /$ IgM, derived from assay of immunoglobulins in vitreous humour, subretinal fluid, and serum from a group of 19 such patients, suggest a lack of free, two-directional, fluid movement across the retinal break. Furthermore the IgG/IgM ratios for the two intraocular fluids were significantly greater than that of serum, this suggesting that these intraocular fluids are formed, at least in part, by a selective transudation of serum.
\end{abstract}

The subretinal fluid from human eyes with rhegmatogenous retinal detachment contains various proteins, ${ }^{1-3}$ and there is some evidence to suggest a plasma derivation for some components of this fluid. ${ }^{245}$ By immunochemical methods soluble proteins from vitreous humour have been identified in human subretinal fluid. ${ }^{3}$ The subretinal fluid also contains lipoproteins, probably derived either locally from degradation of retinal components ${ }^{6}$ or possibly by enzymatic degradation of $\alpha_{1}$ lipoproteins by phospholipase $A$ in the subretinal fluid. ${ }^{7}$ Other investigators



Department of Ophthalmology, St Thomas's Hospital, London SE1 7EH G E Rose

B $M$ Billington

A H Chignell

Correspondence to: $\mathrm{Mr} \mathrm{G} \mathrm{E}$ Rose, Moorfields Eye Hospital, City Road, London ECIV 2PD.

Accepted for publication 7 September 1989
(B)

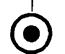

(B) Unidirectional bulk flow; two fluids are significantly different. have proposed on cytological and biochemical evidence that subretinal fluid is derived from retinal elements, from vitreous humour, and from serum leaking from choroidal and retinal vessels.$^{8}$ If vitreous humour and subretinal fluid were derived from multiple sources and no exchange occurred across the retinal hole, the two fluids might be expected to differ (Fig 1A).

Although most evidence is compatible with a net movement of fluid through the retinal hole in a direction from the vitreous humour to the subretinal space (Fig 1B), there remains doubt as to the degree of mixing of the two fluids by this route. In a study of monkey eyes with longstanding rhegmatogenous retinal detachment, fluorescein isothiocyanate-dextran (molecular weight 70000 daltons) was shown to leave the vitreous cavity by bulk flow into the subretinal space ${ }^{8}$ though egress from the latter space was very slow, tracer molecules still being present after six weeks.

If vitreous humour and subretinal fluid reach chemical equilibrium, either by diffusion of solutes (Fig 1C) or by a bulk, two-directional exchange of fluid (albeit with unequal flux in either direction; Fig 1D), then the two fluids should display biochemical similarity.

In order to assess the degree of identity or dissimilarity of the two fluids, we present the first reported comparison of paired specimens of vitreous and subretinal fluid obtained during pars plana vitrectomy for rhegmatogenous retinal detachment.

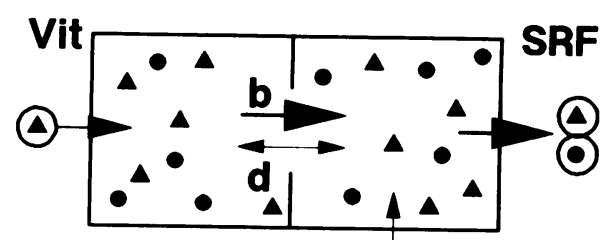

(C)
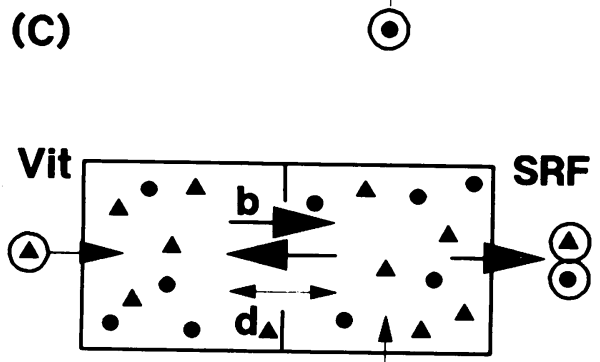

(D)

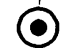

Figure 1: Movement through the retinal holes, between the vitreous humour (Vit) and the subretinal fluid (SRF), in eyes with rhegmatogenous retinal detachment; this movement being by diffusion $(d)$ or by bulk flow of fluid (b).

(A) No movement across hole; fluids in the two compartments are significantly different.

(C) Unidirectional bulk flow and bidirectional diffusion; two fluids are not significantly different.

(D) Bidirectional bulk flow and diffusion; two fluids are not significantly different. 
TABLE I Measured concentrations (mean, standard deviation in brackets, and range) of immunoglobulins $G, A$, and $M(m g / l)$ in specimens of serum, subretinal fluid and vitreous humour from 19 patients with rhegmatogenous retinal detachment

\begin{tabular}{llll}
\hline & \multicolumn{2}{l}{ Immunoglobulin } & \\
\cline { 2 - 4 } Type of fluid & $G$ & $A$ & $M$ \\
\hline Serum & $10790(2080)$ & $2675(957)$ & $1168(537)$ \\
& $7140-15500$ & $1330-4420$ & $585-2680$ \\
Subretinal fluid & $1246(1860)$ & $186(283)$ & $29(42)$ \\
& $7 \cdot 6-6550$ & $2 \cdot 3-1110$ & $3 \cdot 1-162$ \\
Vitreous fluid & $(11 \cdot 6 \%(4 \cdot 02 \%))^{\star}$ & $(7 \cdot 2 \%(2 \cdot 28 \%))^{\star}$ & $(2 \cdot 7 \%(0 \cdot 90 \%))^{\star}$ \\
& $124(118)$ & $22(30)$ & $6 \cdot 7(6 \cdot 3)$ \\
& $2 \cdot 4-397$ & $2 \cdot 0-133$ & $2 \cdot 0-27$ \\
& $(1 \cdot 2 \%(0 \cdot 24 \%))^{\star}$ & $(0 \cdot 97 \%(0 \cdot 37 \%))^{\star}$ & $(0 \cdot 69 \%(0 \cdot 15 \%))^{\star}$ \\
\hline
\end{tabular}

The vitreous humour is liable to dilutional error, the subretinal fluid specimens less so. Values marked thus ${ }^{\star}$ denote mean percentage of the serum concentrations (with standard error of the mean).

An assay of the concentrations of immunoglobulins G (molecular weight approximately 150000 daltons), A (MW 180000), and M (MW $900000)$ was performed and the concentrations compared with those in the serum of the same patients. These molecules were chosen because of their normal presence and stability in biological fluids and their range of large molecular weights, with an assumed poor passage across intact retina.

\section{Patients and methods}

Fourteen male and five female patients who required pars plana vitrectomy and drainage (internal or external) of subretinal fluid were selected for the study; two of the 19 were diabetic. Their ages were from 14 to 75 years (mean 52 years), and the duration of their symptoms of retinal detachment was from one to 52 weeks (mean 11 weeks). There were no cases of giant retinal tear, the largest hole being less than $6 \mathrm{~mm}$ in length and the maximum number of holes recorded being five. Although six of the patients had previous external buckling for retinal detachment in the same eye (three without drainage of subretinal fluid and three with drainage), this was performed at least four weeks prior to the study, a time sufficient to re-establish an equilibrium within the eye.

All patients underwent pars plana vitrectomy. The first sample from the vitreous cavity was aspirated with the Ocutome unprimed to prevent dilution of this specimen within the aspiration tube. The vitreous sample was drawn at a threeway tap system and subretinal fluid was collected either internally, by using a drainage needle passed through the retinal break, or by external drainage (collected within a syringe). When the specimens had been collected, the retinal reattachment procedure was completed by established surgical techniques.

A specimen of blood was drawn at the time of surgery and the serum separated. The three specimens for each patient were stored at $4^{\circ} \mathrm{C}$ until analysis. The concentrations of immunoglobulins $G, A$, and $M$ (IgG, $\operatorname{IgA}$, and $\operatorname{Ig} M)$ in each of the specimens was measured by a rate nephelometric immunoassay (standard reagents, on a Beckman ICS Analyser II; Beckman Instruments, Fullerton, California).

Although every effort was made to avoid dilution of the specimens of intraocular fluids during their collection, this was not possible in all cases. This dilution renders a comparison of
TABLE II Concentration ratios (mean, standard error of mean in brackets, and range) of the lower molecular weight immunoglobulins (classes $G$ and $A$ ) with respect to the higher molecular weight immunoglobulin $M$ in specimens of serum, subretinal fluid, and vitreous humour

\begin{tabular}{lll}
\hline & \multicolumn{2}{c}{ Immunoglobulin concentration ratios } \\
\cline { 2 - 3 } Type of fluid & IgG/IgM ratio & IgA/IgM ratio \\
\hline Serum & $11 \cdot 0(1 \cdot 2)$ & $2 \cdot 9(0 \cdot 5)$ \\
& $3 \cdot 2-20 \cdot 1$ & $0 \cdot 9-7 \cdot 5$ \\
Subretinal fluid & $71 \cdot 2(28 \cdot 2)$ & $7 \cdot 7(2 \cdot 5)$ \\
& $0 \cdot 6-519 \cdot 3$ & $0 \cdot 2-42 \cdot 3$ \\
Vitreous fluid & $22 \cdot 1(4 \cdot 8)$ & $3 \cdot 0(0 \cdot 6)$ \\
& $0 \cdot 6-68 \cdot 8$ & $0 \cdot 3-8.9$ \\
\hline
\end{tabular}

Ratios are independent of any dilutional errors occurring during the collection of the specimens.

measured concentrations of the substances in the vitreous humour and the subretinal fluid (Table I) inaccurate, and the assayed concentrations are not considered further in this paper.

Various indices, independent of the degree of dilution, were derived to overcome the problems of dilution of the specimens during collection. These indices were based on the ratios of concentrations of smaller immunoglobulins (IgG and IgA) to those of the larger immunoglobulin M.

Similar ratios for $\operatorname{IgG} / \operatorname{IgM}$ (or $\operatorname{IgA} / \operatorname{IgM}$ ) in paired specimens of vitreous humour and subretinal fluid would be strongly suggestive of either a common origin for, or a free twodirectional mixing of, the two fluids (Figs $1 \mathrm{C}$ and 1D). Markedly different ratios would suggest an additional source (or sources) for one or both of the fluids as well as a lack of bidirectional mixing (Figs 1A, 1B).

\section{Results}

The concentration ratios (dilution independent) of $\operatorname{IgG} / \operatorname{IgM}$ and $\operatorname{IgA} / \operatorname{IgM}$ in the three fluids are presented in Table II. There is a significant difference between the $\mathrm{IgG} / \mathrm{IgM}$ ratios of serum and vitreous humour (paired Student $t$ test, $\mathrm{p}<0.05$ ) and between the $\mathrm{IgG} / \mathrm{IgM}$ ratios of serum and subretinal fluid $(p<0.05)$. The difference in concentration ratios between vitreous humour and subretinal fluid is, however, not significant. There was no significant difference between the $\operatorname{IgA} / \operatorname{IgM}$ concentration ratios in vitreous humour, subretinal fluid, or serum.

Vitreous humour concentration ratios did not show a significant correlation with those of the subretinal fluids either for $\operatorname{IgG} / \operatorname{IgM}(r=-0.232)$ or for $\operatorname{IgA} / \operatorname{IgM}(r=-0.256)$.

There was no significant difference between the various vitreous humour or subretinal fluid concentration ratios in the six eyes with preoperative uveitis and the other 13 eyes (Table III). The IgG/IgM and IgA/IgM ratios in subretinal fluid had a significant positive linear correlation with the duration of retinal detachment $(r=0.73, p<0.01$ and $r=0.62, p<0.01$, respectively), but vitreous humour concentration ratios did not show this phenomenon. The vitreous humour or subretinal fluid concentration ratios in seven eyes with detachments of one month's duration or less were not, however, significantly different as compared with six eyes with detachments of three months' duration or longer (Table III). 
TABLE III Immunoglobulin concentration ratios (mean, standard error of mean in brackets) in two intraocular fluids, with respect to the presence of preoperative anterior uveitis or the duration of retinal detachment

\begin{tabular}{|c|c|c|c|c|}
\hline & \multicolumn{2}{|c|}{ Vitreous humour } & \multicolumn{2}{|c|}{ Subretinal fluid } \\
\hline & $\operatorname{Ig} G / \operatorname{Ig} M$ & $\operatorname{IgA} / \operatorname{Ig} M$ & $I g G / I g M$ & $\operatorname{Ig} A / \operatorname{Ig} M$ \\
\hline \multicolumn{5}{|c|}{ Preoperative anterior uveitis } \\
\hline Absent (13) & $23 \cdot 1(6 \cdot 54)$ & $3.52(0.76)$ & $76 \cdot 3(40 \cdot 8)$ & $8 \cdot 26(3 \cdot 50)$ \\
\hline Present (6) & $19 \cdot 8(6 \cdot 00)$ & $1.93(0.36)$ & $60 \cdot 4(21 \cdot 8)$ & $6.48(2 \cdot 34)$ \\
\hline \multicolumn{5}{|c|}{ Duration of retinal detachment } \\
\hline $\begin{array}{l}<1 \text { month }(7) \\
>3 \text { months }(6)\end{array}$ & $\begin{array}{l}21 \cdot 5(7 \cdot 26) \\
12 \cdot 4(6 \cdot 65)\end{array}$ & $\begin{array}{l}2.79(0.75) \\
1.35(0.41)\end{array}$ & $\begin{array}{r}46 \cdot 6(18 \cdot 4) \\
145(83 \cdot 3)\end{array}$ & $\begin{array}{r}5 \cdot 31(1 \cdot 86) \\
13 \cdot 2(7 \cdot 27)\end{array}$ \\
\hline
\end{tabular}

\section{Discussion}

Experimental evidence suggests that ocular fluid dynamics in eyes with rhegmatogenous retinal detachment are abnormal. Aqueous humour production is directed posteriorly into the vitreous humour, from which space protein and fluid pass through the retinal hole into the subretinal space; fluid is pumped from this latter space by the retinal pigmentary epithelium, with sequestration of larger molecules within the subretinal space. ${ }^{9}$ Although interpretation is complicated by the possible dilution during collection of the specimens in the present investigation, the markedly greater concentrations of immunoglobulins in subretinal fluid as compared with vitreous humour accord with this hypothesis.

The high values for the average concentration ratios of $\operatorname{IgG} / \operatorname{IgM}$ in vitreous humour and subretinal fluid, both significantly different from the ratio in serum, would suggest that the two intraocular fluids gain a significant contribution either by a selective filtration of serum (with a relative restriction of the passage of the larger IgM molecule), by a selective secretion or local production of IgG within the eye, or by a selective clearance of the large IgM molecule from the eye. Although the $\operatorname{IgG} / \operatorname{IgM}$ and $\operatorname{IgA} /$ IgM ratios in subretinal fluid increased significantly with the duration of retinal detachment, suggesting an increased leakage or a local production of $\operatorname{IgG}$, it is notable that preoperative uveitis did not appear to lead to the increase in the $\mathrm{IgG} / \mathrm{IgM}$ ratio of intraocular fluids that might be expected if either inflamed intraocular vessels leaked the smaller IgG molecule or there was local production of IgG within the eye.

The similar values for IgA/IgM ratios in the three fluids would suggest that somewhat different mechanisms apply to the larger IgA molecule.

On the basis of a simple comparison of average concentration ratios in vitreous humour and subretinal fluid, a common origin for the two intraocular fluids cannot be excluded because the ratios are similar. However, the lack of direct correlation of the ratios in the two fluids implies a 'functional barrier' to maintain such a lack of correlation. Although the lack of correlation might arise from non-representative sampling of each fluid, this is unlikely because of the relatively large samples taken at surgery. Such a functional barrier suggests that the retinal break does not allow a significant diffusion (Fig 1C) or a significant, two-directional, bulk communication (Fig 1D) of the two intraocular fluids. Viewed in the light of surgical manipulation, which can act only to increase mixing of the two fluids, this finding of a restriction of movement across the retinal break becomes even more probable. It is possible that this restrictive, functional 'barrier' exists particularly in those eyes with relatively long-standing retinal detachments, as in the present series of eyes.

Evidence from the present investigation, indicating that free mixing of fluids in the vitreous cavity and the subretinal space does not occur across the retinal hole in eyes with rhegmatogenous retinal detachment, is in agreement with the accepted hypothesis that net movement across the retinal hole occurs from the vitreous humour to the subretinal fluid (Fig 1B). Moreover, such sequestration in the two compartments would accord with the appearance of 'Schleren', passing from the subretinal into the preretinal space, when a retinal hole is disturbed during vitrectomy.

The authors gratefully acknowledge the assay of immunoglobulins performed by Professor A H S Rahi and his staff at the Institute of Ophthalmology.

1 Heath H, Beck TC, Foulds WS. Chemical composition of subretinal fluid. Brf Ophthalmol 1962; 46: 385-96.

2 Chignell AH, Carruthers M, Rahi AHS. Clinical, biochemical and immunoelectrophoretic study of subretinal fluid. $\mathrm{Br} \mathcal{7}$ Ophthalmol 1972; 55: 525-32.

3 Cooper WC, Halbert SP, Manski WJ. Immunochemical analysis of vitreous and subretinal fluid. Invest Ophthalmol Vis Sci 1963; 2: 369-77.

4 Spieser P. Immunoelektrophores der retroretinalen Flüssigkeit. Ophthalmologica 1964; 147: 291-6.

5 Akhmeteli LM, Kasavina BS, Petropavlovskaja GA. Biochemical investigation of the subretinal fluid. $\mathrm{Br} f$ Ophthalmol 1975; 59: 70-7.

6 Lam K-W, van Heuven AJ, Ray S. Lipoproteins in human subretinal fluids. Arch Ophthalmol 1980; 98: 1847-9.

7 Gebhardt DOE, Hogeweg M, Post M. The lipoprotein profile and phosopholipase activity of subretinal fluid. Exp Eye Res 1983; 36: 381-8.

8 Pederson JE, Cantrill HL. Experimental retinal detachment. V. Fluid movement through the retinal hole. Arch Ophthalmol 1984; 102: 136-9.

9 Kaufman PL, Podos SM. The subretinal fluid in primary rhegmatogenous retinal detachment. Surv Ophthalmol 1973; 18: 100-16. 